\title{
Post-Epidural Headache: How Late Can It Occur?
}

\author{
Brian V. Reamy, $M D$
}

Background: Complications of labor epidural anesthesia include a post-dural puncture headache (PDPH). A 2003 meta-analysis described the onset of PDPH as occurring from 1 to 7 days after the procedure. Presented here is the first published case of a PDPH occurring 12 days postpartum.

Methods: Twelve days after an uncomplicated labor epidural a patient was awakened by a "crushing" postural headache. The initial diagnosis was "possible subarachnoid hemorrhage." Lumbar puncture and computed tomography angiogram were normal. Despite medications a severe postural headache persisted and she was referred for an epidural blood patch. Consultants felt the headache onset after 7 days made PDPH impossible. Ultimately a delayed EBP was performed with immediate resolution of her headache.

Discussion: Meta-analyses describe that parturients have a $1.5 \%$ risk of accidental dural puncture during epidural placement. Onset of the headache occurs as early as 1 or as late as 7 days after the procedure. Epidural blood patch is the most effective treatment for PDPH and a rapid response is diagnostic.

Conclusion: Described is the first reported case of a PDPH occurring well outside the normal range of onset 1 to 7 days after epidural anesthesia. The delayed diagnosis and treatment of PDPH in this patient illustrates the limitations of over-rigorous application of pooled analyses to the care of individual patients. (J Am Board Fam Med 2009;22:202-205.)

Lumbar epidural anesthesia is the most common modality used for pain relief during labor in the United States. ${ }^{1}$ When properly performed this technique is safe for the mother and fetus, providing pain relief without the sedative and disorienting effects of narcotic analgesics. The sensation and motor function of the perineum and legs remain intact for delivery, but if needed the anesthesia can be extended to provide a full perineal blockade. The complications of epidural anesthesia have been well described and include hypotension, anesthetic toxicity or allergy, high or spinal anesthesia, infection, neurologic injury and post-dural puncture

This article was externally peer reviewed.

Submitted 28 March 2008; revised 12 June 2008; accepted 17 June 2008.

From the United States Air Force and the Department of Family Medicine, Uniformed Services University, Bethesda, MD.

Funding: none.

Conflict of interest: This paper reflects the views of the author and does not reflect the views of the Uniformed Services University, the USAF or the Department of Defense.

Corresponding author: Brian B. Reamy, MD, Colonel, USAF, Associate Professor and Chair, Department of Family Medicine, Uniformed Services University, 4301 Jones Bridge Road, Bethesda, MD 20814 (E-mail: breamy@ usuhs.mil). headache (PDPH). ${ }^{1}$ An authoritative 2003 metaanalysis definitively described that the onset of PDPH occurs from 1 to 7 days after the procedure. ${ }^{2}$ Presented here is the first published case of a PDPH occurring 12 days postpartum.

\section{Case}

A 33-year-old gravida 2 and para $1\left(\mathrm{G}_{2} \mathrm{P}_{1}\right)$ had a spontaneous vaginal delivery at $363 / 7$ weeks of gestation. The labor progressed for more than 4 hours and anesthesia was provided by an uncomplicated and effective epidural blockade with a 25 gauge needle. No dural puncture was noted at the time of the procedure and the patient reported excellent analgesia during delivery. The patient recovered normally and was feeling well without any headache at hospital discharge 2 days after delivery. The patient had no history of sinusitis, migraine, or other headache syndromes and was otherwise healthy. She reported having an uncomplicated labor epidural during her first delivery 2 years earlier. She was a nonsmoker and had not been consuming caffeine during the pregnancy.

Twelve days postpartum, she was awakened at 0100 by a "crushing" occipital headache. The headache was accompanied by significant nausea and 
was noted to be markedly worse with sitting or standing. The patient denied neck stiffness, visual changes, or any other neurologic symptoms. She presented to the emergency department; the initial diagnostic impression was "possible subarachnoid hemorrhage." Normal vital signs were recorded. A noncontrast head computed tomography scan was interpreted as normal. A lumbar puncture was performed. Tube 1 had clear spinal fluid with normal chemistries, 4 white blood cells, and 93 red blood cells. Tube 4 contained no white blood cells or organisms and 58 red blood cells. Although the emergency physician felt a traumatic tap was the likely source of the blood, he elected to perform a computed tomography angiogram given the clinical symptoms. The angiogram was evaluated as normal. The patient was treated with intravenous fluids, ketorolac, morphine, and metoclopramide and discharged in the care of her husband. The emergency department discharge diagnosis was "headache."

The patient returned to the emergency department the next day with a continued severe postural headache that increased from 1 of 10 to 10 of 10 in severity with any attempt to sit or stand. Her husband reported that she was having difficulty breastfeeding and providing care for the newborn. She was treated with intravenous caffeine and referred for an epidural blood patch (EBP) to treat a possible atypical PDPH. The consulting anesthesiologist, referencing a 2003 meta-analysis, declined to do an EBP and felt that a PDPH was not possible given that the onset of the headache had been more than 7 days after the procedure. He referred the patient to the neurology department; they concurred that the late onset of the headache made PDPH unlikely and prescribed sumatriptan and oral magnesium for a diagnosis of migraine headache.

Later that same day the patient's husband called to report that the patient could still not sit upright because of a severe headache and was prostrate in bed. A second opinion was arranged with a different neurologist who, citing the patient's "classic postural symptoms," diagnosed a PDPH and referred her for an immediate EBP. The EBP was performed and her headache immediately improved from 10 of 10 in intensity to 2 of 10 , and to 0 of 10 the following morning. The headache never recurred and the patient went on to have a normal postpartum clinical course.
Table 1. Symptoms of Post-Dural Puncture Headache

Positional or postural headache
Severe headache
Neck and shoulder stiffness
Photophobia
Nausea
Difficulty in visual accommodation
Diplopia
Dizziness
Tinnitus
Hearing loss

\section{Discussion}

A PubMed search using the keywords "epidural," "complications," and "post-dural puncture headache" yielded 338 citations that were reviewed for applicability to the case described. Meta-analyses described that parturients have a $1.5 \%$ risk of accidental dural puncture with epidural anesthesia. ${ }^{2,3}$ A large retrospective study of over 65,348 anesthetic interventions, from the National Obstetric Anesthetic Database in the United Kingdom, cited the incidence of PDPH as ranging from $1.1 \%$ to $1.9 \% .^{4}$ Of patients who have an accidental dural puncture, $52.1 \%$ will develop a PDPH. ${ }^{2}$ Risk for $\mathrm{PDPH}$ is reduced by using smaller diameter (25- or 27 -gauge) atraumatic needles and by having an experienced operator. ${ }^{3,4}$

PDPH can occur as early as 1 day and as late as 7 days after the procedure. ${ }^{2,4}$ No cases have previously been reported beyond 7 days postpartum. The cardinal features of PDPH are a postural or positional headache that is worsened by sitting or standing and improved by laying supine $(80 \%$ of patients), a "severe" headache that limits activity ( $75 \%$ of patients), and neck/shoulder stiffness ( $43 \%$ of patients); photophobia and nausea are also common with PDPH. ${ }^{2,4}$ Difficulty in accommodation, diplopia, dizziness, tinnitus, and hearing loss are less common, but possible symptoms ${ }^{5,6}$ (Table 1).

The symptoms of PDPH seem to result from the loss of cerebrospinal fluid volume that triggers secondary cerebral vasodilatation and traction on pain sensitive structures of the cerebral contents when assuming an upright position. The differential diagnosis of a PDPH includes nonspecific headache, migraine, cerebral vein thrombosis, sinusitis, meningitis, caffeine withdrawal headache, drug-induced headache, eclampsia, and intracerebral bleeding ${ }^{2,5-7}$ (Table 2). 
Table 2. Differential Diagnosis of Post-Dural Puncture Headache
Caffeine withdrawal headache
Cerebral vein thrombosis
Cerebral tumor
Drug-induced headache
Eclampsia
Intracerebral bleed
Meningitis
Migraine
Non-specific headache
Post-dural puncture headache
Sinusitis

Evidence from the literature supports EBP with 15 to $25 \mathrm{~mL}$ of the patient's own blood as the most effective treatment for PDPH. ${ }^{6,8,9}$ A rapid response to an EBP is both diagnostic and therapeutic. One study reported that, although immediate relief occurred in almost all patients, long-term pain relief was achieved in only $61 \%$ of patients. ${ }^{10}$ It is not entirely clear how an EBP works. An increase in cerebrospinal pressure or reflex cerebral vasoconstriction are likely explanations. ${ }^{11}$ Other forms of treatment include bed rest, intravenous fluids, intravenous caffeine, theophylline, sumatriptan, adrenocorticotropic hormone, gabapentin, and analgesics. ${ }^{12-17}$ Studies support that a recumbent position, intravenous fluids, analgesics, and intravenous caffeine provide some symptom relief for most patients. ${ }^{9-13}$ At present, theophylline, sumatriptan, adrenocorticotropic hormone, and gabapentin have more limited data to support their use. All of these treatments are less effective than EBP. ${ }^{9-11}$

This case is significant for 2 reasons. First, the delayed diagnosis and treatment of PDPH in this patient illustrates the limitations of over-rigorous application of pooled analyses to the care of individual patients. Second, this is the first reported case of PDPH occurring more than 7 days after the procedure. The patient in this case fit all the criteria for a PDPH except for the expected time of onset. Her headache was clearly postural in nature, severe enough to impair breastfeeding and force her to lay prostrate in bed. Other differential diagnostic possibilities were unlikely based on her symptoms and extensive laboratory and radiologic evaluation. She had no fever, normal blood pressure and urinalysis, a negative computed tomography scan and angiogram, no resolution of her headache with analgesics or antimigraine treatments, and immediate resolution of her headache with an EBP. The fact that the onset of her headache was 12 days postpartum, well outside the 1 to 7 day range described in previous meta-analyses, led to a delay in her treatment.

\section{Conclusions}

Described here is the first reported case of a PDPH occurring 12 days after epidural anesthesia. Because $\mathrm{PDPH}$ can occur well after hospital discharge it is important that patients and community physicians are knowledgeable of the signs, symptoms, and optimum treatment modalities for PDPH. This will minimize a delay in treatment during the critical early postpartum period, when breastfeeding is established and mother-child bonding is initiated. Early referral for an EBP will also reduce the severe pain and discomfort experienced by the new mother.

The delayed diagnosis and treatment of PDPH in this patient also illustrates the limitations of over-rigorous application of pooled analyses to the care of individual patients. Although helpful in assisting clinical decision making, meta-analyses can sometimes cause physicians to exclude diagnoses even in the face of compelling individual patientoriented data.

\section{References}

1. Hawkins JL, Goetzl L, Chestnut DH. Obstetric anesthesia. In: Gabbe S, editor. Obstetrics: normal and problem pregnancies. Philadelphia: W.B. Saunders Company; 2007:403-13.

2. Choi PT, Galinski SE, Takeuchi L, Lucas S, Tamayo C, Jadad AR. PDPH is a common complication of neuraxial blockade in parturients: a metaanalysis of obstetrical studies. Can J Anesth 2003;50: 460-69.

3. Halpern S, Preston R. Postdural puncture headache and spinal needle design: meta-analyses. Anesthesiology 1994;81:1376-83.

4. Chan TM, Ahmed E, Yentis SM, Holdcroft A, Obstetric Anaesthetists' Association, NOAD Steering Group. Postpartum headaches: summary report of the National Obstetric Anaesthetic Database (NOAD), 1999. Int J Obstet Anesth 2003;12:107-12.

5. Kuczkowski KM. The management of accidental dural puncture in pregnant women: what does the obstetrician need to know? Arch Gynecol Obstet 2007; 275:125-31.

6. Rutter SV, Shields F, Broadbent M, Popat M, Russell R. Management of accidental dural puncture in labour with intrathecal catheters: an analysis of 10 
years' experience. Int J Obstet Anesth 2001;10:17781.

7. Bleeker CP, Hendriks IM, Booij LH. Postpartum post-dural puncture headache: is your differential diagnosis complete? Br J Anesth 2004;93:461-4.

8. Speigel JE, Tsen LC, Segal S. Requirement for and success of epidural blood patch after intrathecal catheter placement for unintentional dural puncture. Anesthesiology 2001;94:A76.

9. Sandesc D, Lupei MI, Sirbu C, Plavat C, Bedreaq O, Vernic C. Conventional treatment or epidural blood patch for the treatment of different etiologies of post-dural puncture headache. Acta Anaesthesiol Belg 2005;56:265-9.

10. Taivaninen T, Pitkanen M, Touminen M, Rosenberg PH. Efficacy of epidural blood patch for postdural puncture headache. Acta Anesthesiol Scand 1993;37:702-05.

11. Coombs DW, Hooper D. Subarachnoid pressure with epidural blood patch. Reg Anesth 1979;4:3-6.
12. Yücel A, Ozyalçin S, Talu GK, Yücel EC, Erdine S. Intravenous administration of caffeine sodium benzoate for postdural puncture headache. Reg Anesth Pain Med 1999;24:51-4.

13. Camann WR, Murray RS, Mushlin PS, Lambert DH. Effects of oral caffeine on postdural puncture headache. Anesth Analg 1990;70:181-4.

14. Schwalbe SS, Schiffmiller WM, Marx GF. Theophylline for postdural puncture headache. Anesthesiology 1991;75:A1082.

15. Hodgson C, Roitberg HA. The use of sumatriptan in the treatment of postdural puncture headache. Anesthesia 1997;52:808.

16. Kshatri AM, Foster PA. ACTH infusion as a novel treatment for postdural puncture headache. Reg Anesth 1997;22:432-4.

17. Lin YT, Sheen MJ, Huang ST, et al. Gabapentin relieves postdural puncture headache-a report of two cases. Acta Anaesthesiol Taiwan 2007;45:47-51. 